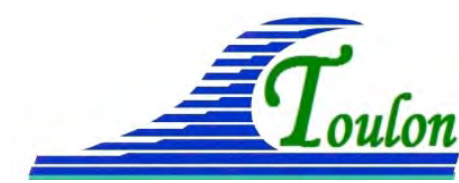

XIV èmes Journées Nationales Génie Côtier - Génie Civil Toulon, 29 juin au $1^{\text {er juillet } 2016}$

DOI:10.5150/jngcgc.2016.032 @ Editions Paralia CFL

disponible en ligne - http://www.paralia.fr - available online

\title{
Rvthme sédimentaire et événements géodynamique plio- quaternaire du bassin côtier du Rharb (Maroc) : apport de la géophysique
}

\author{
Abdelaziz MRIDEKH ${ }^{1}$, Mohamed EL BOUHADIOUI ${ }^{1}$, Bouchaib MAGRANE ${ }^{2}$, \\ Bouabid EI MANSOURI ${ }^{1}$, Jamal CHAO ${ }^{1}$, Malika KILI ${ }^{1}$, Imane AL MAZINI ${ }^{1}$
}

1. Laboratoire Géosciences des ressources naturelles. Département de Géologie. Faculté des Sciences, Université Ibn Tofaïl, Kénitra, Maroc.

abdelaziz.mridekh@gmail.com

2. Agence du bassin hydraulique du Sebou-Kenitra, Maroc

magraneb@yahoo.fr

\section{Résumé :}

Le bassin côtier du Rharb s'étend entre la ville Rabat et la ride de Lalla Zahra sur une distance de $100 \mathrm{~km}$. Il est limité au Nord et au Sud le domaine rifain instable et au NE par le domaine mésétien "relativement stable". Les dépôts du plio-quaternaire sont, pour l'essentiel, fluviatiles, estuariens et alluviaux. Le présent travail a pour objectif de reconstituer, moyennant les outils géophysiques, leur évolution spatiotemporelle et les critères qui augmentent leur vulnérabilité. Selon le modèle établi, la géométrie de ces dépôts se présente en deux configurations, la première sous la dépendance du soulèvement de la méséta, la seconde sous celle de l'avancement, depuis le Miocène moyen, des rides prérifaines. Ces dépôts sont représentés par trois niveaux géoélectriques très discontinus au Nord alors qu'ils sont organisés en électroséquences au Sud. Le croisement des données géophysiques permet de mettre en évidence les zones préférentielles d'intrusion saline, la principale est celle du couloir de Oulad Mahdi. Ces nouveaux résultats permettent d'ouvrir de nouvelles perspectives à l'étude du cadre sédimentaires des dépôts plio-quaternaires, à l'évaluation de leur ressources en eau et à l'étude des effets des aménagements futurs sur les ressources de subsurface.

Mots-clés : Rharb, Plio-quaternaires, Géophysique, Intrusion saline, Ressources en eau, Aménagements.

\section{Introduction}

Le bassin côtier du Rharb (de superficie environ $1750 \mathrm{~km}^{2}$ ) s'étend sur une bande rectangulaire long de $100 \mathrm{~km}$ et large de $25 \mathrm{~km}$, entre la ville de Rabat au Sud et la ride de Lalla Zahra (Moulay Bousselham) au Nord (Figure 1). Par sa position géographique et ses fortes potentialités hydriques la zone d'étude est actuellement le siège d'une forte activité agricole et un dynamisme croissant lié à la mise en place des différents projets structurants (Autoroute, LGV Casablanca-Tanger, barrage de garde sur Sebou et le futur port Kenitra atlantique, etc.). Or, ces infrastructures rencontrent des problèmes 


\section{Thème 2 - Dynamique sédimentaire}

géotechniques, les plus important sont le gonflement des argiles dans les sols hydromorphes (cas de la LGV, autoroute) et l'avancée des sables marins et éoliens (cas du port Kénitra atlantique). En outre, ce domaine est à la croisée de plusieurs dispositifs géodynamiques dont dépendent étroitement la durabilité des aménagements prévus (MRIDEKH, 2015). Le bassin du Rharb est, ainsi, situé sur la terminaison sud occidentale du sillon sud rifain (CHALOUAN \& MICHARD, 2004), zone complexe structurée par une mosaïque de failles régionales et de structures plicatives de diverses directions (ZOUHRI et al., 2004) (Figure 1). Les plus important sont, les failles de Jebha, Fès-Taza-Tainest (CHALOUAN \& MICHARD, 2004 ; BARGACHE, 2011; MRIDEKH, 2015), les structures de Lalla Zahra et le front sud rifain (FLINCH, 1993), et des structures résiduelles tel l'anticlinale de Sidi Yahia (FLINCH, 1993) (Figure 1). Dans le bassin côtier, les structures qui se sont mis en place sont d'orientation globale NW-SE (Figure 1). Dans le bassin offshore, des études récentes ont mis en évidence un nouveau cadre sismostratigraphique et une évolution paléogéographique marquée par le développement de paléodeltas (LE ROY et al., 2014). Deux paléogolfs ont aussi été mise en évidence : celui de Kénitra et de Lalla Zahra (CIRAC, 1985) (Figure 1).Dans les diverses études effectuées dans la zone d'étude, le seul modèle géologique utilisé est basé sur les données de forages hydrogéologiques (COMBE, 1975), modèle devenu insuffisant pour rendre compte de la complexité de mise en place des corps sédimentaires et de leur évolution géodynamique.

L'objectif du présent travail est de présenter les premiers éléments d'un nouveau modèle géodynamique expliquant les diverses configurations du remplissage sédimentaire, condition de base d'une exploitation durable des ressources de cet important espace.

\section{Cadre géologique}

Du point de vue de l'architecture de subsurface, le bassin côtier renferme des structures anticlinales et synclinales en réponse aux mouvements géodynamiques récents du sillon sud rifain (CHALOUAN \& MICHARD, 2004; MRIDEKH, 2015). Ce sont essentiellement les structures anticlinales de Mograne/Oulad Slama, le couloir d'Oulad El Mahdi et les structures associées à la ride de Lalla Zahra (FLINCH, 1993 ; Figure 1). La lithostratigraphie du bassin côtier du Rharb est composé d'une épaisse série de marnes grises d'âge mio-pilocène, dite "marnes bleues", à laquelle font suite des dépôts à caractère régressif, peu épais (COMBE, 1975) du Quaternaire se présentant sous deux lithofaciès. Dans la zone côtière, ils sont formés de sédiments à influence marine, liés aux transgressions quaternaires (Calabrien - Sicilien) (COMBE, 1975). A l'intérieur du continent, les dépôts du Quaternaire sont composés de séries où alternent de manière complexe des sédiments grossiers (galets et cailloutis) et fins (limons et argiles).Vers la limite Est de la zone côtière avec la plaine du Rharb et sur les bordures de l'oued Sebou, 


\section{XIV ${ }^{\text {èmes }}$ Journées Nationales Génie Côtier - Génie Civil \\ Toulon, 29 juin au $1^{\text {er }}$ juillet 2016}

les terrains sont représentés par des faciès argileux ou limoneux correspondant aux alluvions du Quaternaire terminal (Rharbien) (LE COZ, 1964).

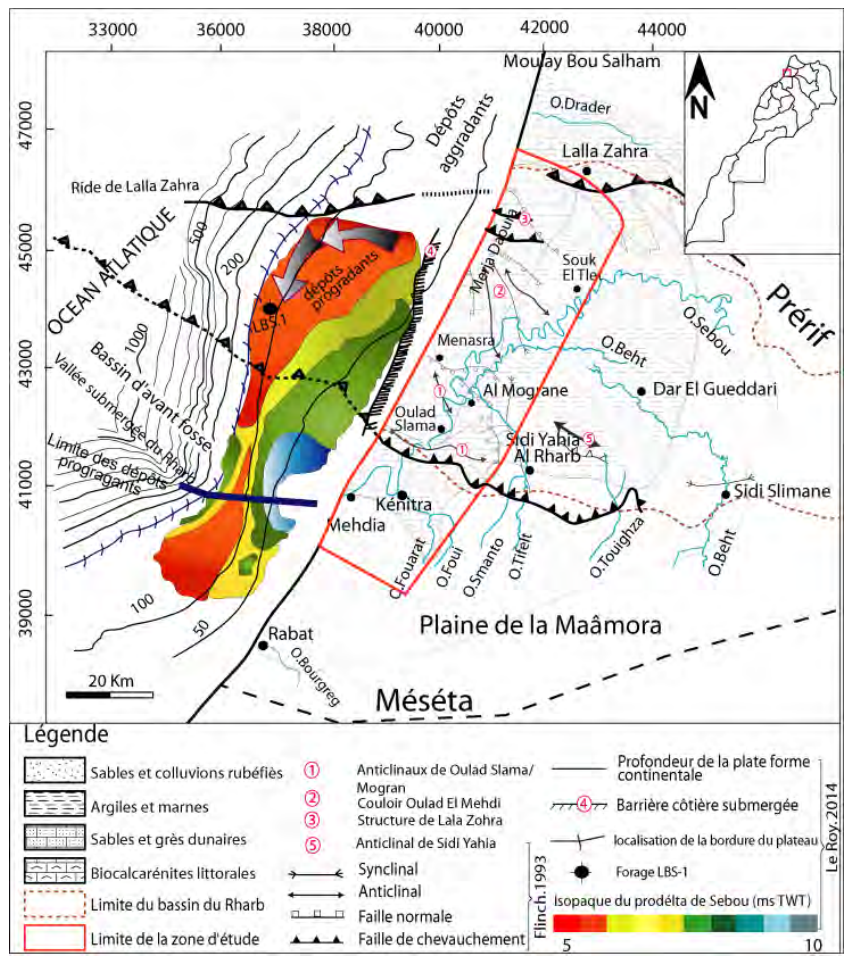

Figure1. Carte de situation, paléogéographie et structure de subsurface de la zone d'étude (compilée d'après les travaux de CIRAC, 1985 ; FLINCH, 1993 ; LE ROY et al., 2014 et nos propres observations dans le domaine onshore).

Les ressources en eau sont localisées au niveau d'aquifères gréseux. Elles souffrent d'une forte exploitation par un millier de puits et de forages, aggravée par une nette augmentation de la superficie irriguée. En conséquence, il a été observé une nette variation piézométrique dans les forages de la nappe de Menasra (EL MANSOURI, 1999) alors que les piézomètres situés au voisinage de la ligne de côte présentent une stabilité du niveau d'eau. La salinité des eaux de la nappe a connu une forte augmentation, les valeurs de la salinité de 1964 étaient homogènes sur l'ensemble de la nappe avec des valeurs inférieures à 1 g/l, alors que celles de 2007 elles présentent des nodules autours de $2 \mathrm{~g} / \mathrm{l}$.

\section{Base de données et méthodologie}

Dans la présente étude, nous utilisons une base de données compilée sous SIG comportant 50 forages hydrogéologiques dont 30 possèdent des enregistrements diagraphiques (Fig. 2). Une diagraphie est un enregistrement en continu des paramètres physiques : résistivité, radioactivité naturelle ou induite, densité, sonique et polarisation. 


\section{Thème 2 - Dynamique sédimentaire}

Elles servent, entre autres, à la détermination de la lithologie et à l'établissement de corrélations (KELLY \& MARES, 1993). Les données électriques utilisées sont les sondages électriques verticaux (SEV). Plus de $500 \mathrm{SEV}$ ont été analysés. Cette méthode est basée sur la mesure du potentiel électrique à partir de l'injection d'un courant électrique dans le sous-sol. (TELFORD et al., 1990). L'interprétation des S.E.V n'est pas unique en raison des principes connus d'"équivalence" et de "suppression". De nombreux calages aux sondages mécaniques sont effectués.

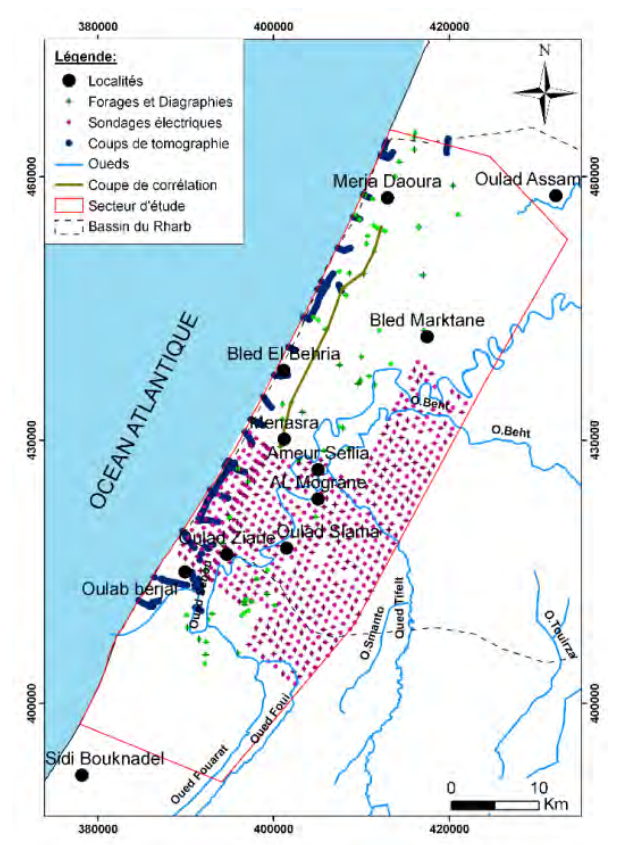

Figure 2. Localisation des SEV dans la zone d'étude.

\section{Résultats}

A l'issu de l'analyse et de l'interprétation des différents documents géophysiques et des données des forages hydrogéologiques, nous présentons les principaux résultats suivants :

\subsection{Géométrie du substratum}

Les cartes des figures 3a et 3b mettent en évidence le rapport entre les structures profondes du bassin côtier, la géométrie du substratum marneux et l'allure des isopièzes. Ainsi, la profondeur du substratum marneux montre deux familles de valeurs de part et d'autre d'une ligne de direction subéquatoriale (Fig. 3a). Au Nord, les isovaleurs du substratum sont orientées NE-SW et se distribuent de part et d'autre d'une zone haute parallèle à la ligne de côte. Au Sud, par contre, ces isovaleurs présentent un gradient régulier dénotant un passage progressif depuis le continent vers la plateforme 


\section{XIV ${ }^{\text {èmes }}$ Journées Nationales Génie Côtier - Génie Civil \\ Toulon, 29 juin au $1^{\text {er }}$ juillet 2016}

continentale. Les isopièzes semblent être influencées par cette architecture, le même découpage de part et d'autre d'une ligne subéquatoriale peu y être observé.

a
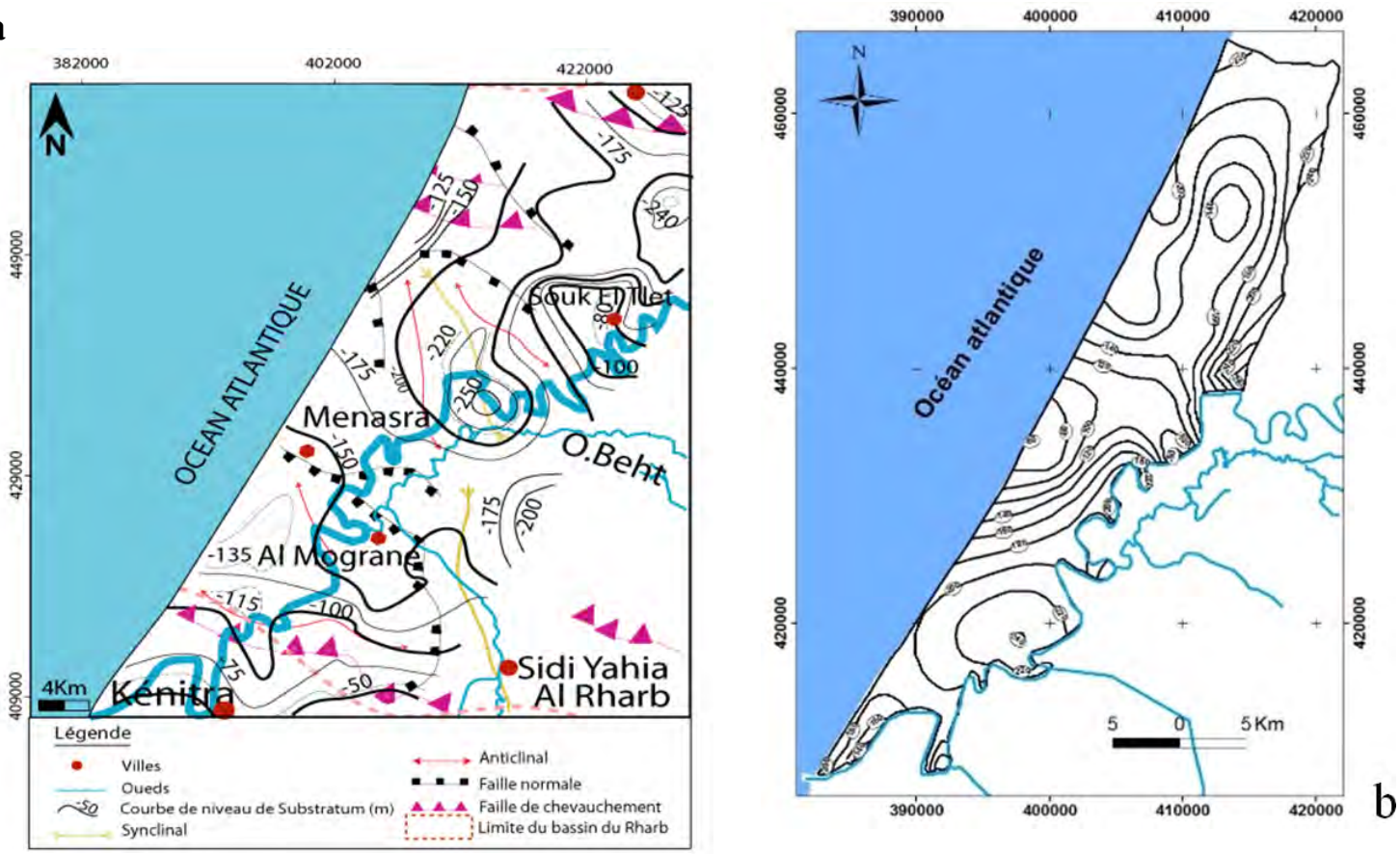

Figure 3. Carte du substratum marneux (EL BOUHADDIOUI et al., 2014) (a) et du niveau piézométrique de la nappe de Menasra (LPEE, 2007)(b).

\subsection{Nouveau cadre électro-stratigraphique}

Afin de mettre en évidence l'impact de la structuration du substratum sur l'évolution sédimentaire du bassin côtier, une coupe de corrélation diagraphique a été établie (Figure 4). Elle montre que les électrofaciès diagraphiques se présentent en trois ou quatre niveaux géoélectriques, s’organisant en deux grands groupes de part et d'autre d'une discontinuité (Figure 4) :

Le premier groupe montre des électrofaciès dentelés de forme "cylindrique" (passage abrupt à la base et au sommet), généralement de forme chenalisée à la base, alors qu'au niveau des profondeurs $-100 \mathrm{~m}$, les électrofaciès montrent une géométrie ondulée très probablement en relation avec la croissance de la structure de Lalla Zahra située plus au nord (figure 4).

Le deuxième groupe, situé au Sud de la discontinuité électrique, montre plutôt que les électrofaciès s'organisent en électrobancs réguliers électriquement progressivement résistant ayant une allure d'électroséquences régulières (figure 4). 


\section{Thème 2 - Dynamique sédimentaire}

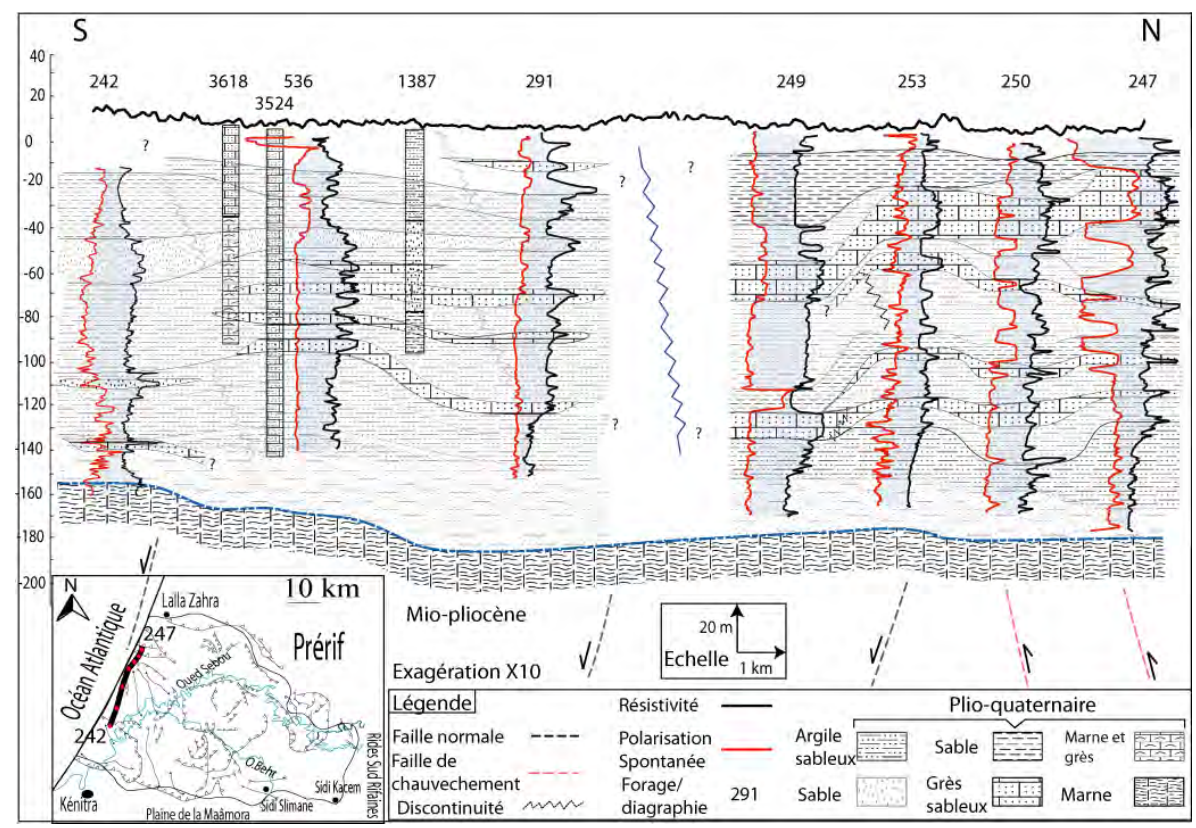

Figure 4.Coupe de corrélation NNE-SSW des électrofaciès diagraphiques à travers le bassin côtier.

\subsection{Imagerie géophysique de l'intrusion saline}

Le caractère hydrogéologique de la nappe de Menasra, partie intégrante du bassin côtier (Figure 1), tient à la rencontre à l'intérieure même du réservoir souterrain, d'eaux douces continentales avec des eaux marines. Cet équilibre fragile s'établit et s'exprime par l'existence d'une zone de mélange généralement peu épaisse et de géométrie variable que nous avons tenté de suivre à travers l'établissement des coupes de tomographie électriques.

Ainsi, l'inversion du profil Oulad Mehdi II-4 (Figure 5) montre des valeurs de résistivités très élevées (supérieur à $300 \Omega$.m) (plages dominantes rouges dans les sections) caractérisant les sables et probablement les grès secs, des valeurs moyennes(de 30 à $120 \Omega$.m) (plages vertes à jaunes) caractérisant les sables et grès sous nappe et des valeurs très faibles (plages bleu de résistivité inférieur à $10 \Omega . m$ ). Cette coupe est habillée par un enregistrement diagraphique mettant en évidence le niveau où la valeur de résistivité subi une chute importante. L’interface eau douce-eau salée est ainsi située à une profondeur de $80 \mathrm{~m}$ en 2007. La comparaison de cette diagraphie avec un enregistrement effectué en 1958, où l'interface ED/ES était à $124 \mathrm{~m}$, permet de mettre en évidence clairement sa remontée. L'exploitation de nombreuses données de tomographie électrique similaire permet de montrer que l'intrusion au niveau de la région d'Oulad Mehdi est l'une des plus importantes, sa situation par rapport au couloir tectonique du même nom permettrait de lier les intrusions salines à la structure profonde (Figures 1 \& 5). 


\section{XIV vèmes Journées Nationales Génie Côtier - Génie Civil \\ Toulon, 29 juin au $1^{\text {er }}$ juillet 2016}

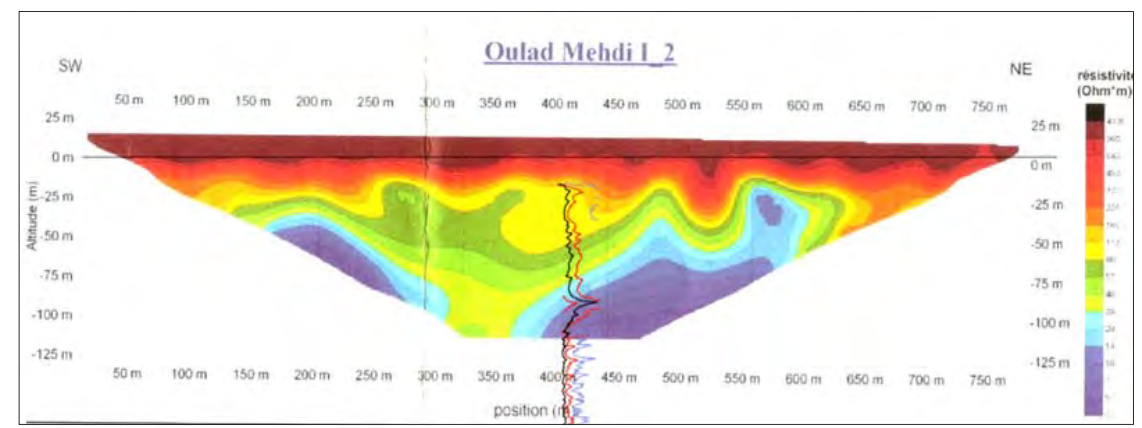

Figure 5.Tomographie électrique et coupe de diagraphie (log de résistivité électrique, 16 " et 64") indiquant une chute brutale de la résistivité témoin d'une augmentation de la salinité.

\section{Conclusion}

Le bassin côtier du Rharb constitue l'abouchement de la nappe du Rharb vers l'Océan atlantique. Il se situe sur une zone d'intersection de dispositifs géodynamiques complexes : la meseta, les rides prérifaines et se trouve actuellement l'objet de grands projets de génie civil.

Dans le présent travail, on propose de nouveaux éléments de la structure de subsurface et de son cadre électro-stratigraphique, et ce dans le but de contraindre la géométrie des dépôts et des aquifères, approche indispensable à la gestion optimale des ressources naturelles. Les données géophysiques présentées ont, ainsi, permis d'établir les éléments d'un nouveau cadre électro-litho-stratigraphique de la zone. Trois niveaux géoélectriques, surmontant le niveau marneux de base (généralement très conducteur) sont mis en évidence. Du Sud au Nord, les électrofaciès deviennent plus discontinus, du fait, très probablement, de la prédominance de la tectonique plio-quaternaire. Leur organisation "séquentielle" au Sud serait l'expression de l'influence du niveau marin. L’imagerie géophysique permet, d'autre part, de mettre en évidence l'existence de zones préférentielles d’intrusion marine, l'exemple présenté est celui du couloir d'Oulad Mahdi où la confrontation d'un enregistrement diagraphique à une coupe de tomographie électrique a permis la localisation précise de l'interface eau douce-eau salée.

Ces nouveaux résultats permettent de rendre compte des risques de déséquilibre que peuvent subir les ressources de subsurface, à moyen et à long terme, dans le cas où des mesures spécifiques ne sont pas prises lors des dimensionnements des nouvelles infrastructures. A ce titre, la construction du port Kénitra atlantique et de la ligne de la LGV (en plus de l'accroissement rapide de la zone industrielle "Kénitra atlantique") constitueront un stress supplémentaire, que seul un suivi par application d'approches pluridisciplinaires (mise en place de salinomètres, mesure régulière par tomographie, rationalisation de l'extraction des matériaux et suivi par imagerie satellitaire) permettra de réduire les risques auxquels sont confrontés les écosystèmes et les ressources de surface et de subsurface. 


\section{Références bibliographiques}

CHALOUAN A., MICHARD A. (2004). The Alpine Rif Belt (Morroco): A case of Montain Bulding in a Subduction - Transformation Fault Triple Junction. Pure and Applied Geophysics, Vol. 161(3), pp 489-519. http://dx.doi.org/10.1007/s00024-003-2460-7

CIRAC P. (1985). Le bassin sud-rifain occidental au Néogène supérieur. Evolution de la dynamique sédimentaire et de la paléogéographie au cours d'une phase de comblement. Thèse d'état, Université Bordeaux I, France, 283 p.

COMBE M. (1975). Le bassin Rharb-Maâmora et les petits bassins septentrionaux des oueds Dradère et Soueire. In : Ressources en eaux du Maroc. T2. Plaines et Bassins Du Maroc Atlantique, Ed. Serv. Géol., pp 93-128.

EL BOUHADDIOUI M., MRIDEKH A., KILI M., EL MANSOURI B., EL GASMI E., MAGRANE B. (2014). Le Bassin du Rharb, Répartition des lithofacièsplioquaternaires, contexte paléogéographique et géodynamique, contribution des diagraphies. Notes et Mém, Serv, Géol, Maroc, n 577, pp 125-137.

EL MANSOURI B. (1999). Développement d'outils et concepts pour la gestion des eaux souterraines. Applications à l'aquifère côtier du Rharb. Thèse d'état ès Science, Université Ibn-Tofail, Faculté des Sciences, Kénitra, 142 p.

FLINCH J.F. (1993). Tectonic evolution of the Gibraltar arc. PhD Thesis, Rice University, Houston, 381 p.

KELLY W.E., MARES S. (1993). Applied Geophysics in Hydrogeological and Engineering Practice. Elsevier Science Publishers B.V, Amesterdam, 285 p.

LE COZ J. (1964). Le Rharb, Fellahs et colons. Étude de géographie régionale, Tome 1, Les cadres de la nature et de l'histoire, Rabat (482 p., 83 fig., 23 pl. phot.).

LE ROY P., SAHABI M., MAAD N., RABINEAU M., GUTSHER M.-A., BABONNEAU N., VAN VLIET LANOE B., AIT BRAHIM L., M'HAMMDI N., TRENTESAUX A., DAKKI M., HSSAIN M. (2014). 3D architecture of Quaternary sediment along the NW Atlantic Moroccan Rharb continental shelf: A stratal pattern under the dual control of tectonics and climatic variations. Marine and Petroleum Geology, Vol. 49, pp 129-142. http://dx.doi.org/10.1016/j.marpetgeo.2013.10.003

MRIDEKH A. (2015). Géodynamique récente de quelques bassins ouest marocains. Implication hydrogéologique et pétrolière. Mémoire HDR. Université Ibn Tofail. Kénitra. 300 p.

TELFORD W.M., GELDART L.P., SHERIFF R.E. (1990). Applied geophysics. $2^{\text {nd }}$ edition, Cambridge University press, 770 p. http://dx.doi.org/10.1017/cbo9781139167932

ZOUHRI L., GORINI C., MANIA J., DEFFONTAINES B., ZEROUALI A. (2004). Spatial distribution of resistivity in the hydrogeological systems, and identification of the catchment area in the Rharb basin, Morocco. Hydrological Sciences Journal, Vol. 49(3), pp 387-398. http://dx.doi.org/10.1623/hysj.49.3.387.54350 